\title{
Triplet State EPR Spectra
}

\author{
R. C. StevenSON* \\ Biophysics Research Division, University of Michigan, Ann Arbor, Michigan 48109
}

Received June 27, 1983; revised September 29, 1983

\begin{abstract}
A characterization of triplet EPR spectra in terms of the eight phenomenological parameters of the spin Hamiltonian is given. Closed-form solutions for the three possible resonant fields between triplet energy levels are presented, and thus the calculation is valid over a wide range of spin-spin interaction strengths. The importance of the halffield $(g \approx 4)$ spectra for extracting information about the Hamiltonian parameters is demonstrated, and a recipe is presented for fitting triplet EPR data using computergenerated spectra.
\end{abstract}

\section{INTRODUCTION}

Triplet EPR spectra reflect the strong spin-spin interaction between two spin-1/2 species. The spectroscopist may use the parameters which characterize the spin-spin interaction to infer information about the paramagnetic site, such as interaction distance or the relative orientation of the interacting species-information which could suggest chemical bonding, for example.

The study of triplet systems has a long history so this paper will not present any new fundamental results. It does demonstrate how to obtain more information from triplet EPR spectra than was realized previously. Reference (l) contains an extensive list of papers on triplet systems. Included in the theoretical discussions is a list of special cases where the resonant fields reduce to relatively simple expressions. In Ref. (2), the triplet eigenvalues are obtained in the same manner as presented here. In Ref. (3) the eigenvalue problem is formulated in an invariant geometric notation similar to that below. Reference (4) presents some computer simulations for a spin-1 EPR spectra except that the Hamiltonian is not as general as here.

The triplet Hamiltonian $\mathscr{H}_{\text {TRIP }}$ discussed below assumes all field-independent interactions arise from electronic spin interactions. One could imagine adding to the Hamiltonian an applied field-independent term proportional to spin, say $\mathbf{C} \cdot \mathbf{S}$. This term would imply that there is some fixed magnet within the molecule. For instance, the vector $\mathbf{C}$ could be proportional to the average magnetic field due to nuclear spins within and around the electron paramagnetic site. This paper assumes that regardless of how $\mathbf{C} \cdot \mathbf{S}$ may arise, it is negligible compared to the other terms in the Hamiltonian.

A spin-1 triplet system is, mathematically, the simplest spin system above spin 1/2: one can readily perform a closed-form diagonalization of the $3 \times 3$ Hamiltonian

* Presently at Bell Telephone Laboratories, Incorporated, 1600 Osgood Street, North Andover, Mass. 01845. 
matrix operator. Furthermore, from the closed-form expressions for the energy levels, the paper derives closed-form expressions for resonant fields. These expressions are cumbersome for hand calculations, but they are quite reasonable to implement for a computer calculation.

There is also a discussion of the results of computer experiments, performed with a computer program called TRIPLET, into the behavior of triplet spectra with respect to the Hamiltonian parameters. These simulations reveal the importance of the halffield (or $g 4$ ) data to the spectroscopist. One may obtain, without simulation, good initial estimates of the principal triplet $g$ values from the $g 4$ data if the investigator has $g 4$ data at two frequencies. Also, the $g 4$ lineshape can be a sensitive indicator of the relative orientation of the principal axis system of the spin-spin interaction tensor and the principal axes of the $g$ tensor. The $g=2$ (or $g 2$ ) data reflects the strength of the spin-spin interaction, i.e., the diagonal elements of the spin-spin interaction. The $g 4$ intensity offers a check on the estimate for the largest diagonal element, since the $g 4$ intensity goes as the square of that diagonal element. These estimates of the diagonal elements of the spin-spin interaction, coupled with the information contained in the $g 4$ data, allow one to extract all Hamiltonian parameters after only a few simulations.

This procedure for fitting triplet data restricts a possibly lengthy search through a parameter space of 11 variables (8 Hamiltonian parameters and 3 linewidth variables). The reader is directed to Ref. (5) for an alternative approach to computer simulations of triplet data using a least-squares type of fitting algorithm.

\section{THE METHOD}

For EPR powder spectra calculations, one must perform the following sequence of steps:

(a) Choose a suitable spin Hamiltonian.

(b) Calculate the energy eigenvalues and eigenvectors; i.e., diagonalize the spin Hamiltonian.

(c) Calculate transition rates between states. This process involves

(i) solving the resonance condition for the resonant fields, and

(ii) finding the matrix elements of the magnetic dipole transition operator.

(d) Sum over all possible orientations of the spin system with respect to the applied magnetic field to produce a powder spectrum.

These steps are outlined below for the spin-1 Hamiltonian.

\section{Step (a): Defining the Parameters of the Triplet Hamiltonian}

The physical model assumes that there are two unpaired spins, $\mathbf{S}_{\mathrm{a}}$ and $\mathbf{S}_{\mathrm{b}}$, at sites $a$ and $b$ within a molecule and that these two spins represent spin- $1 / 2$ species. In addition to the unpaired spin angular momentum at each site, there are also $g$ tensors, $G_{a}$ and $G_{b}$, (assumed to be symmetric) associated with each spin. Reference (6) discusses the case for an asymmetric $g$ tensor. Because these are electronic systems, each site has a magnetic moment. For sites $a$ and $b$

$$
\boldsymbol{\mu}_{\mathrm{a}}=-\beta \mathbf{G}_{\mathrm{a}} \cdot \mathbf{S}_{\mathrm{a}}, \quad \text { and } \quad \boldsymbol{\mu}_{\mathrm{h}}=-\beta \mathbf{G}_{\mathrm{h}} \cdot \mathbf{S}_{\mathrm{b}} .
$$


The energy of interaction of the two magnetic moments with an applied magnetic field $\mathbf{B}$ is the Zeeman interaction $\mathscr{H}_{0}$, where

$$
\mathscr{H}_{0}=-\mu_{\mathrm{a}} \cdot \mathbf{B}-\mu_{\mathrm{b}} \cdot \mathbf{B}
$$

or, expressed as the sum of a symmetric term and an antisymmetric term,

$$
\mathscr{H}_{0}=\beta\left(\mathbf{S}_{\mathrm{a}}+\mathbf{S}_{\mathrm{b}}\right) \cdot \frac{\left(\mathbf{G}_{\mathrm{a}}+\mathbf{G}_{\mathrm{b}}\right)}{2} \cdot \mathbf{B}+\beta\left(\mathbf{S}_{\mathrm{a}}-\mathbf{S}_{\mathrm{b}}\right) \cdot \frac{\left(\mathbf{G}_{\mathrm{a}}-\mathbf{G}_{\mathrm{b}}\right)}{2} \cdot \mathbf{B}
$$

The two spins may also interact through a tensor $\mathbf{J}$ (here of unspecified origin). This spin-spin interaction is

$$
\mathscr{H}_{1}=\mathbf{S}_{\mathrm{a}} \cdot \mathbf{J} \cdot \mathbf{S}_{\mathrm{b}}
$$

$\mathbf{J}$ is a rank 2 Cartesian tensor (nine degrees of freedom) and, one may decompose $\mathbf{J}$ into the sum of its three irreducible parts.

$$
\begin{array}{cl}
\mathbf{J} \equiv-2 J 1 & \begin{array}{l}
\text { transforms like a scalar under a } \\
\text { one-dimensional representation of } 0^{+}(3)
\end{array} \\
-2 K \times 1 & \begin{array}{l}
\text { transforms like a vector under a } \\
\text { three-dimensional representation of } 0^{+}(3) \\
\text { (antisymmetric term) }
\end{array} \\
+ \text { 2D } & \begin{array}{l}
\text { transforms like a (traceless) symmetric tensor under } \\
\text { a five-dimensional representation of } 0^{+}(3) \text { when the five } \\
\text { independent components are written as a column vector. }
\end{array}
\end{array}
$$

Therefore,

$$
\mathscr{H}_{1}=-2 J \mathbf{S}_{\mathrm{a}}^{\prime} \cdot \mathbf{S}_{\mathrm{b}}+2 \mathbf{K} \cdot\left(\mathbf{S}_{\mathrm{a}} \times \mathbf{S}_{\mathrm{b}}\right)+2 \mathbf{S}_{\mathrm{a}} \cdot \mathbf{D} \cdot \mathbf{S}_{\mathrm{b}}
$$

\begin{tabular}{|c|c|c|}
\hline & State $\left|S, M_{\mathrm{s}}\right\rangle$ & Energy \\
\hline Triplet & $\begin{aligned}|1,1\rangle & =|\uparrow\rangle_{\mathrm{a}} \otimes|\uparrow\rangle_{\mathrm{b}} \\
|1,0\rangle & =\left(|\uparrow\rangle_{\mathrm{a}} \otimes|\downarrow\rangle_{\mathrm{b}}+|\downarrow\rangle_{\mathrm{a}} \otimes|\uparrow\rangle_{\mathrm{b}}\right) / \sqrt{2} \\
|1,-1\rangle & =|\downarrow\rangle_{\mathrm{a}} \otimes|\downarrow\rangle_{\mathrm{b}}\end{aligned}$ & $-J / 2$ \\
\hline Singlet & $\begin{aligned}|0,0\rangle & =\left(|\uparrow\rangle_{\mathrm{a}} \otimes|\downarrow\rangle_{\mathrm{b}}-|\downarrow\rangle_{\mathrm{a}} \otimes|\uparrow\rangle_{\mathrm{b}}\right) / \sqrt{2} \\
|\uparrow\rangle & =\left(\begin{array}{l}1 \\
0\end{array}\right) \quad|\downarrow\rangle=\left(\begin{array}{l}0 \\
1\end{array}\right)\end{aligned}$ & $3 J / 2$ \\
\hline
\end{tabular}

One generally assumes that

$$
|J| \gg|\mathbf{K}| \gg\|\mathbf{D}\| \text {. }
$$

Moriya (7) gives a justification for this ordering.

If $|J|$ satisfies the inequality [7] and $\left\|\mathbf{G}_{a}+\mathbf{G}_{b}\right\| \gg\left\|\mathbf{G}_{a}-\mathbf{G}_{b}\right\|$, total spin is a good quantum number; the basis set with which to expand the Hamiltonians $\mathscr{H}_{0}$ and $\mathscr{H}_{1}$ is the total spin basis set, that is, the eigenfunctions of the scalar spin-spin interaction (see Table 1). One then extracts the triplet Hamiltonian from the $S=1(3 \times 3)$

TABLE 1

Eigenstates and Eigenvalues of SCALAR SPIN-SPIN INTERACTION 
subspace of $\mathscr{H}_{0}$ and $\mathscr{H}_{1}$. Because the $S=1$ basis states are symmetric under interchange of spin, the expectation of any terms antisymmetric under interchange of spin must vanish; only symmetric terms from $\mathscr{H}_{0}+\mathscr{H}_{1}$ remain upon projection onto the $S=1$ subspace. The scalar term in $J$ may be dropped under demand of zero trace. Using the spin-1/2 algebra and the traceless and symmetry properties of the tensor D, one may write the triplet Hamiltonian as

$$
\mathscr{H}_{\mathrm{TRIP}} \equiv \beta \mathbf{S} \cdot \mathbf{G} \cdot \mathbf{B}+\mathbf{S} \cdot \mathbf{D} \cdot \mathbf{S} \text {. }
$$

The vector $\mathbf{S}$ is the total spin. Written in matrix form, it becomes

$$
\mathbf{S}=\left.\left(\mathbf{S}_{\mathbf{a}}+\mathbf{S}_{\mathbf{b}}\right)\right|_{s=1}=\left[\begin{array}{ccc}
\hat{\xi}_{0} & \hat{\xi}_{-1} & 0 \\
-\hat{\xi}_{+1} & 0 & \hat{\xi}_{-1} \\
0 & -\hat{\xi}_{+1} & -\hat{\xi}_{0}
\end{array}\right],
$$

where

$$
\hat{\xi} \pm=\mp \frac{(\hat{x} \pm i \hat{y})}{\sqrt{2}} ; \quad \hat{\xi}_{0}=\hat{z},
$$

and $G$ exists in $3 \times 3$ Cartesian space, where

$$
\mathbf{G}=\left(\mathbf{G}_{\mathrm{a}}+\mathbf{G}_{\mathrm{b}}\right) / 2 .
$$

Thus, the triplet Hamiltonian retains only information about the average of the two original $g$ tensors and the weakest portion, generally, of the spin-spin interaction (reduced by a factor of 2).

The triplet $g$ tensor is symmetric since, by assumption, it is the average of two $g$ symmetric tensors. Therefore, $\mathbf{G}$ has three principal values, which define a coordinate frame, called the body frame, attached to the molecule. The diagonal form of $\mathbf{G}$ is

Here,

$$
\left.\mathbf{G}\right|_{\text {Body }} \equiv\left[\begin{array}{lll}
g_{x} & 0 & 0 \\
0 & g_{y} & 0 \\
0 & 0 & g_{z}
\end{array}\right]
$$

$$
g_{x} \leqslant g_{y} \leqslant g_{z} .
$$

One can rewrite the triplet Zeeman interaction

$$
\mathscr{X}_{\text {ZEE }}=\beta \mathbf{S} \cdot \mathbf{G} \cdot \mathbf{B}
$$

as

where

$$
\mathscr{K}_{\mathrm{ZEE}}=g \beta B \mathrm{~S} \cdot \hat{n},
$$

In this case

$$
g=g(\hat{r})=|\mathbf{G} \cdot \hat{r}| .
$$

$$
\hat{r} \equiv \frac{\mathbf{B}}{B} \equiv\left[\begin{array}{l}
1_{x} \\
1_{y} \\
1_{z}
\end{array}\right] \quad \text { and } \quad \hat{n}=\mathbf{G} \cdot \hat{r} / g .
$$

See Fig. 1 for a representation of $\hat{r}$ and $\hat{n}$ in the body frame.

The number of degrees of freedom in the triplet spin-spin or zero-field splitting (ZFS) interaction

$$
\mathscr{H}_{\text {zFs }}=\mathbf{S} \cdot \mathbf{D} \cdot \mathbf{S}
$$




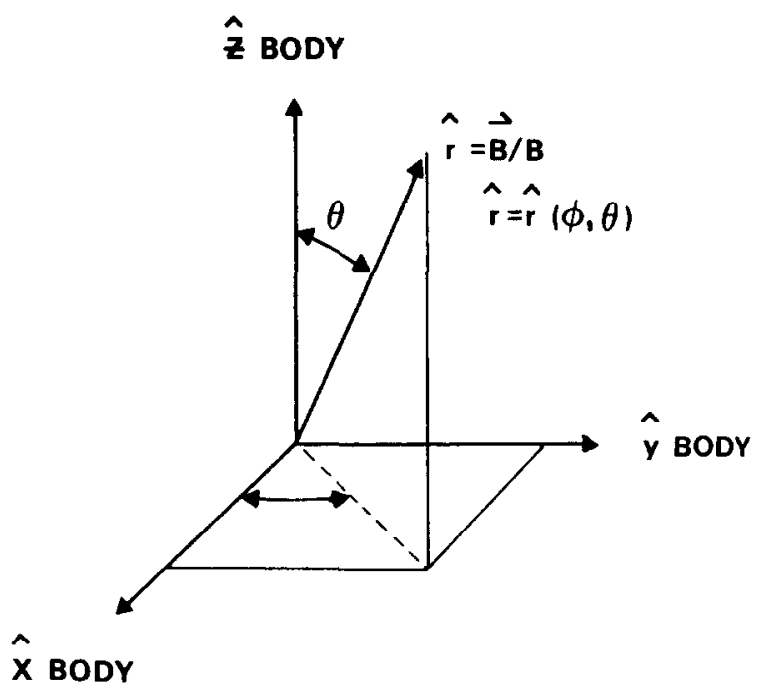

$\hat{z}$ BODY

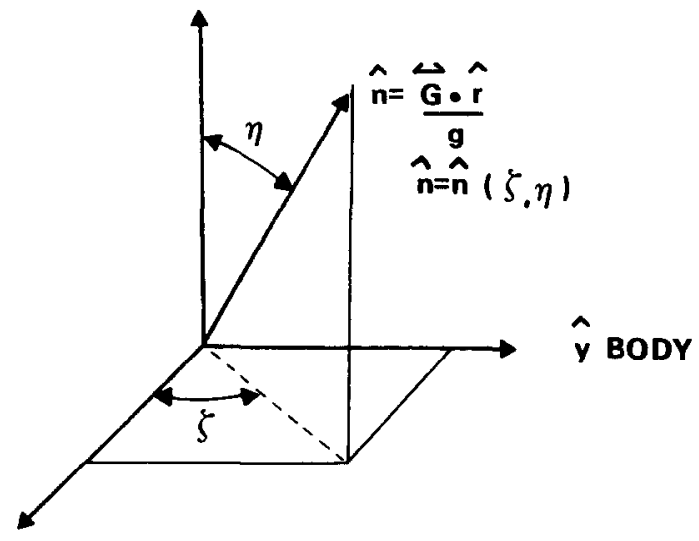

\section{$\hat{x}$ BoDr}

FIG. 1. Representation of unit vectors $\hat{r}$ and $\hat{n}$ in the body frame.

is five, a more reasonable number in terms of the ability to fit parameters than the nine degrees of freedom of $\mathbf{J}$. $\mathbf{D}$, because it is symmetric, also has a diagonal form, $\mathbf{D}_{0}$, where

$$
\mathbf{D}_{0}=\left(\begin{array}{ccc}
X & 0 & 0 \\
0 & Y & 0 \\
0 & 0 & Z
\end{array}\right) .
$$

Note that the $3 \times 3$ matrices of $G$ in Eq. [12] and $D_{0}$ in Eq. [19] exist in Cartesian space and do not operate in the $3 \times 3$ spin space of the matrix of $\mathbf{S}$. Because $D$ is traceless, 


$$
X+Y+Z=0
$$

so that Eq. [19] accounts for two degrees of freedom. One may parameterize the remaining three degrees of freedom by an Euler rotation $R(\alpha, \beta, \gamma)$ of $\mathrm{D}_{0}$ :

$$
\mathrm{D}=R(\alpha, \beta, \gamma) \mathrm{D}_{0} R^{\mathrm{T}}(\alpha, \beta, \gamma) .
$$

One may interpret $R(\alpha, \beta, \gamma)$ as actively rotating $\mathrm{D}_{0}$ from a position where its principal axes are coincident with the body frame axis $\hat{x}, \hat{y}$, and $\hat{z}$ to new positions

$$
\left(\hat{e}_{1}, \hat{e}_{2}, \hat{e}_{3}\right)=R(\alpha, \beta, \gamma)(\hat{x}, \hat{y}, \hat{z}) .
$$

The ZFS interaction has a very simple form imposed upon it by the constraints of hermiticity, tracelessness, and the allowance of only five degrees of freedom. These constraints restrict $\mathscr{H}_{\mathrm{ZFS}}$ to the general form

$$
\mathscr{H}_{\text {ZFS }}=\left(\begin{array}{ccr}
D_{0} & D_{1}^{*} & D_{2}^{*} \\
D_{1} & -2 D_{0} & -D_{1}^{*} \\
D_{2} & -D_{1} & D_{0}
\end{array}\right) .
$$

$D_{0}$ is real and $D_{1}$ and $D_{2}$ are complex. If one were to expand $\mathscr{H}_{\text {zFs }}$ in terms of irreducible tensor operators, $D_{0}, D_{1}^{*}, D_{2}^{*}$ would be proportional to the coefficients of the tensors of rank $0,+1$, and $+2\left(D_{1}\right.$ and $D_{2}$ are proportional to the coefficients of the tensors of rank -1 and -2 ).

All parts of the triplet Hamiltonian have now been specified.

\section{Step (b): Diagonalization of $\mathscr{H}_{\text {TRIP }}$}

To calculate transition rates, one needs to know energy levels and normalized eigenstates of the paramagnetic system. The next step toward the construction of the spin-coupled spectra is the diagonalization of $\mathscr{K}_{\text {TRIP }}$ given in essentially closed form expressions.

The characteristic equation for $\mathscr{H}_{\text {TRIP }}$ is

$$
\left|\mathscr{H}_{\mathrm{TRIP}}-\lambda 1\right|=0,
$$

or

$$
\sum_{n=0}^{3} \operatorname{tr}_{n}\left(\mathscr{H}_{\text {TRIP }}\right)(-\lambda)^{3-n}=0,
$$

where $\operatorname{tr}_{n}\left(\mathscr{H}_{\text {TRIP }}\right)$ is the $n$th generalized trace of $\mathscr{H}_{\text {TRIP }}$. For the cubic polynomial of Eq. [25] these traces are $\operatorname{tr}_{0}=1 ; \mathrm{tr}_{1}=$ ordinary trace; $\operatorname{tr}_{2}=$ sum of all $2 \times 2$ determinants whose diagonal elements are diagonal elements of $\mathscr{K}_{\mathrm{TRIP}} ; \mathrm{tr}_{3}=$ ordinary determinant. The trace of $\mathscr{H}_{\text {TRIP }}$ is zero by design. One can show that the second trace $\operatorname{tr}_{2}$ is

$$
\operatorname{tr}_{2}\left(\mathscr{X}_{\text {TRIP }}\right) \equiv a=-A^{2}+t_{2},
$$

where

$$
A=g \beta B,
$$

and

$$
t_{2} \equiv \operatorname{tr}_{2}\left(\mathscr{H}_{\mathrm{ZFS}}\right)=X Y+X Z+Y Z \text {. }
$$


Thus, the second trace of $\mathscr{H}_{\text {TRIP }}$ factors to a sum of the second trace from Zeeman interaction, plus the second trace of the triplet spin-spin interaction. As for the determinant of $\mathscr{H}_{\text {TRIP }}$, one can show that

$$
\operatorname{det}\left(\mathscr{H}_{\text {TRIP }}\right) \equiv-b=2 D_{0} A^{2}+t_{3},
$$

where

$$
D_{0}=(1 / 2) \hat{n} \cdot \mathbf{D} \cdot \hat{n}
$$

and

$$
t_{3}=\operatorname{det}\left(\mathscr{H}_{\mathrm{ZFS}}\right)=X Y Z \text {. }
$$

By expressing the second trace and the determinant in the manner above, one can organize a somewhat confusing collection of parameters in a manageable form for both evaluation in a computer program and further algebraic manipulation. Because $t_{2}$ and $t_{3}$ are invariants, their values do not change regardless of the orientation of the magnetic field with respect to the spin system. Hence, they need only be evaluated once in the calculation of powder spectra. The characteristic equation then has the form

$$
\lambda^{3}+a \lambda+b=0 .
$$

Because one seeks the real roots of Eq. [32], the trigonometric solution to the cubic equation ( 8 ) is the most convenient form for two reasons: (1) One can readily check the reality of the roots, and (2) the trigonometric solution allows one to solve for the resonance conditions in a straightforward manner. The three roots are

$$
\lambda_{1}=m \cdot \cos (\theta), \quad \lambda_{2}=m \cdot \cos (\theta+4 \pi / 3),
$$

and

$$
\lambda_{3}=m \cdot \cos \left(\theta+\frac{2 \pi}{3}\right)
$$

where

$$
m=2 \sqrt{\frac{-a}{3}} \quad \text { and } \quad \cos (3 \theta)=\frac{3 b}{a m} ; \quad 0 \leqslant \theta \leqslant \pi / 3 .
$$

These roots satisfy the following relations:

$$
\lambda_{1}+\lambda_{2}+\lambda_{3}=0 ; \quad \lambda_{1}>\lambda_{2}>\lambda_{3} ; \quad \lambda_{1}>0 \quad \text { and } \quad \lambda_{3}<0 .
$$

After finding the eigenvalues, one must then calculate the eigenvectors. TRIPLET calculates one solution for the unnormalized eigenvector $\left|u^{(i)}\right\rangle$, corresponding to the eigenvalue $\lambda_{i}$, where

$$
\left|u^{(i)}\right\rangle=\left[\begin{array}{l}
u_{1}^{(i)} \\
u_{2}^{(i)} \\
u_{3}^{(i)}
\end{array}\right]
$$

by finding the pseudoinverse of $\mathscr{H}_{\text {TRIP }}-\lambda_{\mathrm{i}} 1$; i.e.,

$$
u_{j}^{(i)}=\operatorname{cof}_{i j}\left(\mathscr{K}_{\text {TRIP }}-\lambda_{i} 1\right) \text {. }
$$

One must then normalize the set of $\left|u^{(i)}\right\rangle$ with any desired phase. 


\section{Step (c): Transition Rates}

To induce transitions from an initial normalized magnetic state $|i\rangle$ to a final normalized magnetic state $|f\rangle$, the EPR spectrometer applies a polarized microwave field of frequency $\nu$, which couples the magnetic component of that field, with amplitude $B_{1}$, to the magnetic moment $\mu$ of the triplet spin system, where

$$
\boldsymbol{\mu}=-\beta \mathbf{G} \cdot \mathbf{S} \text {. }
$$

This coupling is the magnetic dipole transition operator

$$
\mathscr{H}^{\prime}(t)=\mathscr{H}_{1}^{\prime} \cos (\omega t)
$$

where

$$
\mathscr{H}_{1}^{\prime}=-\boldsymbol{\mu} \cdot \mathbf{B}_{1}
$$

Fermi's golden rule, to a good approximation, gives the (absorption) transition rate $W_{i j}$ as

$$
W_{i f}(\nu)=\frac{2 \pi}{\hbar}\left|\left\langle f\left|\left(\frac{\mathscr{P}_{1}^{\prime}}{2}\right)\right| i\right\rangle\right|{ }^{2} \rho_{\nu}\left(\lambda_{f}-\lambda_{i}-h \nu\right) .
$$

To include all possible transitions, one must sum over initial states. The total frequency space transition rate is

$$
W_{\mathrm{T}}(\nu)=\sum_{i<f} W_{i f}(\nu)
$$

The transition rate has two parts which require nontrivial manipulation to place the two expressions in a form suitable for calculation. They are

(1) the density of final states $\rho_{\nu}$, which contains the resonance condition:

$$
\lambda_{f}-\lambda_{i}-h \nu=0
$$

(2) the magnitude of the matrix element $\left\langle f\left|\mathscr{H}_{1}^{\prime}\right| i\right\rangle$ squared.

Let us consider the density function.

Most EPR spectrometers are field-swept spectrometers, instead of frequency-swept spectrometers; consequently, one needs to transform $\rho_{\nu}$ from frequency space to field space. There is a well-defined procedure for doing this type of transformation from the theory of statistical density functions (9). This procedure, a fundamental theorem of density functions, stated in a nonrigorous form, says that if $f_{y}(y)$ is a density function for a random variable $y$, and $y$ is a function of another random variable $x$,

$$
y=g(x)
$$

then

$$
f_{y}(y)=\sum_{i=1}^{n} \frac{f_{x}\left(x_{i}\right)}{\left|g^{\prime}\left(x_{i}\right)\right|}
$$

where $x_{i}, i=1, \ldots, n$, are the $n$ real roots of Eq. [44].

To convert the density function $\rho$ from frequency space to field space, one must solve the equation

$$
\Delta E_{i f}=h \nu,
$$


which corresponds to Eq. [44], for the resonant field that satisfies this equation. Because there are three possible energy levels, there are three possible transitions:

and

$$
\begin{aligned}
& \lambda_{1}-\lambda_{2}=h \nu=m \sqrt{3} \cos (\Theta+\pi / 6), \\
& \lambda_{2}-\lambda_{3}=h \nu=m \sqrt{3} \cos (\Theta+3 \pi / 6),
\end{aligned}
$$

$$
\lambda_{1}-\lambda_{3}=h \nu=m \sqrt{3} \cos (\theta-\pi / 6) \text {. }
$$

One comes to the expressions in Eq. [47] after applying standard trigonometric identities to the expressions in Eq. [33]. See Fig. 2 for a typical triplet energy-level scheme and the three resonances.

All three resonance conditions in Eq. [47] have the same form, namely,

$$
\cos (\theta+\alpha)=\frac{h \nu}{m \sqrt{3}},
$$

where the angle $\theta$ is a function of the magnetic field: $\theta=\theta(B)$. To find the resonant fields, one must solve Eq. [48] for $B$. Using a multiangle trigonometric identity one may easily show that

$$
\sin ^{2}(3 \theta)=\left(4 \cos ^{3}(\theta+\alpha)-3 \cos (\theta+\alpha)\right)^{2}
$$

is true for $\alpha=-\pi / 6, \pi / 6$, or $3 \pi / 6$. Combining Eqs. [34], [48], and [49], one can show that all three resonance conditions invert (i.e., one can eliminate $\theta$ ) to

$$
4 a^{3}+9 a^{2}(h \nu)^{2}+6 a(h \nu)^{4}+(h \nu)^{6}+27 b^{2}=0 .
$$

Inserting Eqs. [26] and [29] for $a$ and $b$ in terms of $A=g \beta B$, one finds that Eq. [50] reduces to

$$
\left(A^{2}\right)^{3}+p\left(A^{2}\right)^{2}+q\left(A^{2}\right)+r=0 .
$$

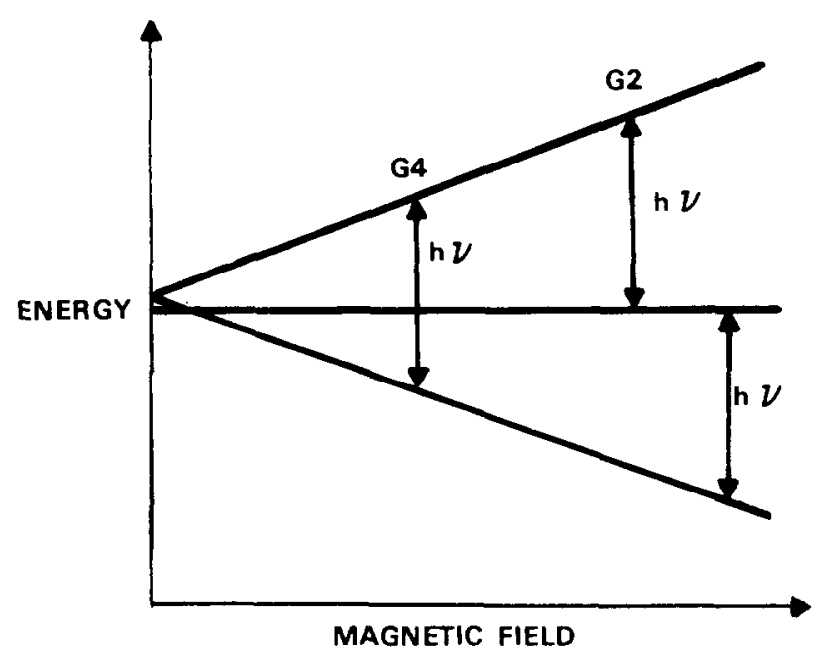

FIG. 2. Typical energy-level diagram for a single-powder orientation and the three corresponding resonances. 
The coefficients of this cubic equation in $A^{2}$, with $\delta^{2}=(h v)^{2}+t_{2}$, are

$$
\begin{aligned}
& p=-\frac{3}{4}\left[3 \delta^{2}+t_{2}\right]-27 D_{0}^{2}, \\
& q=\frac{3}{2}\left[\left(\delta^{2}\right)^{2}+3 t_{2} \cdot \delta^{2}\right]-27 D_{0} t_{3}, \\
& r=\frac{1}{4}\left[\left(\delta^{2}\right)^{3}+3 t_{2} \cdot\left(\delta^{2}\right)^{2}\right]-\frac{27}{4} t_{3}^{2} .
\end{aligned}
$$

Note from Eq. [52] the rather curious fact that the product of the three roots of Eq. [51] is

$$
\left(A_{1} \cdot A_{2} \cdot A_{3}\right)=r,
$$

a number independent of the projection $D_{0}$. The resonant fields occur in the cubic Eq. [51] in $A^{2}$; this situation reflects the fact that the resonance condition is insensitive to which direction the spectrometer applies the magnetic field. To obtain the resonant fields, one should again use the trigonometric solutions to the cubic equation. In this case the equation has a "trace" term $p$, so that all trigonometric solutions must be shifted by $-p / 3$ (1/3 of the trace). Equations [51] and [52] are the only known (to this author) closed-form expressions for the triplet resonant fields.

The next portion of the density function transformation theorem which one must calculate is the renormalization factor $\left|g^{\prime}\left(x_{i}\right)\right|$. In terms of frequency, the resonance condition is

$$
\nu(B)=\Delta E_{i f} / h,
$$

so that the renormalization factors are

$$
\left|g^{\prime}\left(x_{\mathrm{i}}\right)\right| \leftrightarrow\left|\frac{\partial \nu}{\partial B}\right|_{B-B_{i}}, \quad i=1,2 \text {, or } 3 .
$$

The Appendix gives a closed-form expression for these three derivatives. Consequently, the transition rate, for a single species, from frequency space to field space, is

$$
W_{\mathrm{T}}(\nu) \rightarrow W_{\mathrm{T}}(B)=\sum_{r=1}^{3} \frac{2 \pi}{\hbar} \frac{\rho_{B}\left(B_{r}\right)}{\left|\frac{\partial \nu}{\partial B}\right|_{B-B_{r}}}\left|\left\langle f_{r}\left(\frac{\mathscr{E}_{1}^{\prime}}{2}\right) \mid i_{r}\right\rangle\right|^{2} .
$$

Generally, the modeling of $\rho_{B}\left(B_{r}\right)$ is phenomenological; one picks a lineshape that is convenient and gives a good fit to the data. A Gaussian density function is a popular choice; for a Gaussian,

$$
\rho_{B}\left(B_{r}\right)=\frac{1}{\sqrt{2 \pi}} \frac{1}{\sigma_{B_{r}}} e^{-\left(B-B_{r}\right)^{2} /\left(2 \sigma_{B_{r}}\right)^{2}} .
$$

The linewidth or variance $\sigma_{B_{r}}$ is a phenomenological parameter which one must determine experimentally. However, if one assumes that the density function $\rho_{v}(\nu)$ has a variance $\sigma_{\nu}$, independent of frequency, then one can show that the approximate relationship between the variance of the frequency space density function and the field space density function $(9$, p. 152) is 


$$
\sigma_{v}^{2} \approx\left|\frac{\partial \nu}{\partial B}\right|_{B_{r}}^{2} \cdot \sigma_{B_{r}}^{2}
$$

or

$$
\left|\sigma_{B_{r}}\right| \approx \frac{\left|\sigma_{\nu}\right|}{\left|\frac{\partial_{\nu}}{\partial B}\right|_{B_{r}}}
$$

Thus, renormalization also affects the linewidths in the transition from frequency space to field space.

The second part of the transition rate is the matrix element of $\mathscr{H}_{1}^{\prime}$ between an initial and final state. Because

$$
\mathscr{H}_{1}^{\prime}=\beta \mathbf{S} \cdot \mathbf{G} \cdot \mathbf{B}_{1}
$$

then

$$
\left\langle f\left|\mathscr{R}^{\prime}\right| i\right\rangle=\beta\langle f|\mathbf{S}| i\rangle \cdot \mathbf{G} \cdot \mathbf{B}_{1} \equiv \beta \mathbf{S}_{i j^{\prime}} \cdot \mathbf{G} \cdot \mathbf{B}_{1} .
$$

If the components of the eigenvectors, from Eq. [37] say, are

then

$$
|i\rangle=\left[\begin{array}{l}
c_{+1} \\
c_{0} \\
c_{-1}
\end{array}\right]_{\text {Body }} \quad \text { and } \quad|f\rangle=\left[\begin{array}{l}
d_{+1} \\
d_{0} \\
d_{-1}
\end{array}\right]_{\text {Body }}
$$

$$
\left.\mathbf{S}_{i f}\right|_{\text {Body }}=\left[\begin{array}{c}
\frac{1}{\sqrt{2}}\left[\left(c_{+1}+c_{-1}\right) d_{0}^{*}+c_{0}\left(d_{+1}^{*}+d_{-1}^{*}\right)\right] \\
\frac{i}{\sqrt{2}}\left[\left(c_{+1}-c_{-1}\right) d_{0}^{*}-c_{0}\left(d_{+1}^{*}-d_{-1}^{*}\right)\right] \\
c_{+1} d_{+1}^{*}-c_{-1} d_{-1}^{*}
\end{array}\right] .
$$

The standard convention places $\mathbf{B}_{1}$ along the $\hat{x}$ axis of the laboratory coordinate frame, so that in the body frame

$$
\left.\mathbf{B}_{1}\right|_{\text {Body }}=B_{1} R(\phi, \theta, \psi) \hat{x},
$$

where $R(\phi, \theta, \psi)$ is an active three-space rotation. Thus,

where

$$
\left.\left\langle f\left|\mathscr{H}_{1}\right| i\right\rangle\right|_{\text {Body }} ^{2}=\beta^{2} B_{1}^{2} \mathbf{S}_{i f} \cdot \mathbf{G} \cdot\left(R(\phi, \theta, \psi) \hat{x} \hat{x}^{\mathrm{T}} R^{\mathrm{T}}(\phi, \theta, \psi)\right) \cdot \mathbf{G} \cdot \mathbf{S}_{i f}^{*},
$$

$$
R(\phi, \theta, \psi) \hat{\mathrm{x}}=\left[\begin{array}{c}
\cos \phi \cos \theta \cos \psi-\sin \phi \sin \psi \\
\cos \phi \sin \theta \cos \psi-\cos \phi \sin \psi \\
-\sin \theta \cos \psi
\end{array}\right]
$$

If one defines the dyadic $\mathbf{M}(\phi, \theta, \psi)$ to be

$$
M(\phi, \theta, \psi)=R(\phi, \theta, \psi) \hat{x} \hat{x}^{\mathrm{T}} R^{\mathrm{T}}(\phi, \theta, \psi),
$$

then the total transition rate for a single species becomes

$$
W_{\mathrm{T}}(B)=\frac{2 \pi}{h} \frac{\beta^{2} B_{1}^{2}}{4} \sum_{r=1}^{3}\left[\mathbf{S}_{i f^{*}} \mathbf{G} \cdot \mathbf{M} \cdot \mathbf{G} \cdot \mathbf{S}_{i j}^{*}\right]_{r} \frac{\rho_{B}\left(B_{r}\right)}{\left|\frac{\partial \nu}{\partial B}\right|_{B_{r}}}
$$


Step (d): Powder Spectra

EPR samples, especially biological samples, are typically amorphous and one generally ignores magnetic interaction of one molecule with another. Thus, one assumes that the paramagnetic molecules are randomly oriented. For any molecule, the probability density (that the body frame assumes some orientation $\theta, \phi, \psi$ in $d \Omega d \psi$ with respect to the laboratory frame) is constant and equal to $1 /(4 \pi \cdot 2 \pi)$. The expected value $I(B)$ of $W_{\mathrm{T}}(B)$ for $N$ independent molecules is then

$$
I(B)=\frac{N}{(4 \pi)(2 \pi)} \int d \Omega d \psi W_{\mathrm{T}}(B)
$$

In practice, because of field modulation, the spectrometer measures $\partial I / \partial B$. One can perform the integral over the third angle $\psi$ in closed form because the only quantity which depends on $\psi$ in $W_{\mathrm{T}}(B)$ is the dyadic $M(\phi, \theta, \psi)$. A computer must perform the remaining integral over solid angle. Averaging $M(\phi, \theta, \psi)$ over $\psi$ one finds that

$$
\frac{1}{2 \pi} \int_{0}^{2 \pi} d \psi M(\phi, \theta, \psi) \equiv M(\phi, \theta)=1 / 2\left(1-\hat{r} \hat{r}^{\mathrm{T}}\right) .
$$

Therefore, the total triplet powder derivative spectrum is

$$
\frac{\partial I}{\partial B}=\frac{N \beta^{2} B_{1}^{2}}{8 \hbar} \sum_{r=1}^{3} \int d \Omega\left[\mathbf{S}_{i f^{\prime}} \cdot \mathbf{G} \cdot \mathbf{M}(\phi, \theta) \cdot \mathbf{G} \cdot \mathbf{S}_{i j}^{*}\right]_{r} \frac{\partial \rho_{B}(B) / \partial B}{\left|\frac{\partial \nu}{\partial B}\right|_{B_{r}}}
$$

or

$$
\frac{\partial I}{\partial B} \propto \sum_{r=1}^{3} \int d \Omega\left\{\mathbf{S}_{i f} \cdot\left[g^{2}\left(1-\hat{n} \hat{n}^{\mathrm{T}}\right)\right] \cdot \mathbf{S}_{i f}^{*}\right\}_{r} \cdot \frac{\partial \rho_{B}(B) / \partial B}{\left|\frac{\partial \nu}{\partial B}\right|_{B r}}
$$

The FORTRAN program, TRIPLET, calculates $\partial I / \partial B$ according to Eq. [69] (see (10) for a listing of TRIPLET). Because the eigenvalues and eigenvectors of $\mathscr{X}_{\text {TRIP }}$, as well as the resonant fields, have closed-form expressions, the computer program can calculate triplet spectra over a wide range of input parameters.

\section{TYPICAL BEHAVIOR OF $\partial I / \partial B$}

If a triplet EPR computer simulation program is to be useful in modeling data, then one should not blindly search through the parameter space. One needs insight into the behavior of the function $\partial I / \partial B$. The next sections discuss typical behavior of $\partial I / \partial B$ and how the various Hamiltonian parameters manifest themselves in the powder spectra.

If the Zeeman interaction is the dominant interaction, then for fairly accurate expressions for the eigenvalues of $\mathscr{H}_{\text {TRIP }}$, to order $1 / A$,

$$
\begin{aligned}
& \lambda_{1}=A+D_{0}-(1 / 2)\left(t_{2}+3 D_{0}^{2}\right) / A, \\
& \lambda_{2}=2 D_{0},
\end{aligned}
$$


and

$$
\lambda_{3}=-A+D_{0}+(1 / 2)\left(t_{2}+3 D_{0}^{2}\right) / A .
$$

The three resonant conditions, to first order, are

and

$$
\begin{aligned}
& h \nu=\lambda_{2}-\lambda_{3}=A-3 D_{0} \rightarrow B_{1}=\left(h \nu+3 D_{0}\right) /(g \beta), \\
& h \nu=\lambda_{1}-\lambda_{2}=A+3 D_{0} \rightarrow B_{2}=\left(h \nu-3 D_{0}\right) /(g \beta),
\end{aligned}
$$

$$
h \nu=\lambda_{1}-\lambda_{3}=2 A \quad \rightarrow B_{3}=h \nu /(2 g \beta) .
$$

The first two equations of Eq. [71] imply that for each "powder orientation," at normal EPR frequencies, say $\mathrm{X}$ band to $\mathrm{Q}$ band, there are two resonances $B_{1}$ and $B_{2}$, which are split by about $6 D_{0}\left(D_{0}\right.$ in units of gauss). The center of this splitting is approximately at the field value corresponding to $g$, i.e., $h \nu /(g \beta)$. The third resonance, $B_{3}$, is the half-field or $g 4$ resonance. Its position on the field scan is close to midway between zero field and the center of splitting for $B_{1}$ and $B_{2}$. Each of the three resonances depends on the solid angle $\Omega$ only through the dependence on $D_{0}$ and $g$. Note also that the ratio of $\partial \nu / \partial B_{3}$ to $\partial \nu / \partial B_{1}$ or $\partial \nu / \partial B_{2}$ is about 2:1. From Eq. [58], this renormalization implies that the $g 4$ linewidth is about half that of the $g 2$ linewidths.

If the anisotropy of the tensor $D$ is much larger than the $g$ tensor, then the parameter $D_{0}$ dominates the variation of the two $g 2$ resonances with solid angle. A powder $g 2$ spectrum reflects quite plainly the anisotropy of $D$. To see this one strips the expression for the intensity $I(B)$ to its bare essentials, the powder spectrum becomes the sum of many "sticks"; i.e., it is determined only by the resonance condition; therefore,

$$
\left.I(B)\right|_{g 2} \stackrel{\substack{\text { bare } \\ \text { essentials }}}{\sim} \int d \Omega \delta\left(B-B_{1}(\Omega)\right)+\int d \Omega \delta\left(B-B_{2}(\Omega)\right) .
$$

Since the delta function is a density function, one may apply the density function transformation theorem, in a slightly modified form, to evaluate the integrals in Eq. [72] over solid angle. Hence,

$$
\int_{\Omega} d \Omega \delta(f(\Omega))=\int_{d \Gamma} d \Gamma \frac{1}{\left|\nabla_{\Omega} f\right|_{\Gamma}} .
$$

$\Gamma$ is a curve on the $\phi, \theta$ plane, determined by the constraint equation $f(\Omega)=0$. Also,

and

$$
\nabla_{\Omega}=\hat{\theta} \frac{\partial}{\partial \theta}+\hat{\phi} \frac{1}{\sin \theta} \frac{\partial}{\partial \phi}
$$

$$
f(\Omega)=B-B_{i}(\Omega), \quad i=1,2 .
$$

The integrand of Eq. [73] will diverge when

$$
\left|\nabla_{\Omega} B_{i}(\Omega)\right|=0, \quad \mathrm{i}=1,2 .
$$

These poles dominate the appearance of derivative powder spectra. If one refers to the first-order solutions for the $g 2$ resonances and also assumes that the first-order behavior reflects a generally monotonic behavior of $B_{1}$ and $B_{2}$ with respect to $D_{0}$, then $\nabla_{\Omega} B_{i}=0$ when $\nabla_{\Omega} D_{0}=0$, assuming that the anisotropy of $g$ is negligible 
compared to $D_{0}$. The angles $\phi$ and $\theta$ for which $\nabla_{\Omega} D_{0}=0$ are precisely those angles for which $D$ attains its extreme values. Thus, one expects that the integrand of $I(B)$ for a real powder spectrum to diverge whenever, during the powder integration, the angles $\phi$, and $\theta$ are such that $D_{0}=X, Y$, or $Z$. However, the integration over $\Omega$ can reduce the poles from spikes to steps. A finite linewidth rounds the corners of the steps, as well as damps any further poles not reduced by the integration over solid angle. Typically then, the $g 2$ powder spectrum exhibits six turning points or "lines" (two resonances, three lines per resonance) with three corresponding "splittings," called principal splittings, of magnitude (in gauss) of

$$
\left|B_{1}-B_{2}\right| \approx 6|X|, 6|Y| \text {, and } 6|Z| \text {. }
$$

The centers of splittings vary with the Euler rotation $R(\alpha, \beta, \gamma)$. Table 2 gives the approximate $g$ values for the centers; they must lie between $g_{x}$ and $g_{z}$ because by Eq. [13] $g_{x}$ and $g_{z}$ are the extremes of the $g$ tensor

$$
g_{x} \leqslant g_{1}, g_{2}, g_{3}, \leqslant g_{z} .
$$

Note that if in real data $g_{1}, g_{2}$, or $g_{3}$ are not the principal $g$ values, then there must be an Euler rotation $R(\alpha, \beta, \gamma)$. Figure 3 shows a typical absorption and derivative spectra generated by TRIPLET.

Data from the $g 4$ region of triplet spectrum is very important to the spectroscopist because it contains much information about magnitudes of many of the phenomenological parameters. Equation [71] shows that the $g$ tensor dominates the half-field resonant positions to first order. From this approximation, one would expect the $g 4$ resonance to look much like an anisotropic spin-1/2 powder spectrum with three principal turning points determined by the $g$ values. Indeed, these two spectra do have similar features. However, there are important differences due to second-order contributions from $\mathbf{D}$ to the line positions or resonance condition.

Using the second-order expressions for the eigenvalues $\lambda_{1}$ and $\lambda_{3}$, one finds a second-order expression for the $g 4$ resonance:

$$
B_{3} \stackrel{\substack{\text { second } \\ \text { order }}}{=} \frac{h \nu}{2 g \beta}+\frac{\left(t_{2}+3 D_{0}^{2}\right)}{g \beta B_{3}} .
$$

One can show that the second-order correction

$$
\left(t_{2}+3 D_{0}^{2}\right) /\left(2 g B_{3}\right)
$$

TABLE 2

APproximate $g 2$ SPLitTings and the EfFective $g$ Values

\begin{tabular}{lcc}
\hline Splitting & Center of splitting & Direction \\
\hline $6|X|$ & $g_{1}=g\left(\hat{e}_{1}\right)$ & $\hat{e}_{1}=R(\alpha, \beta, \gamma) \hat{x}$ \\
$6|Y|$ & $g_{2}=g\left(\hat{e}_{2}\right)$ & $\hat{e}_{2}=R(\alpha, \beta, \gamma) \hat{y}$ \\
$6|Z|$ & $g_{3}=g\left(\hat{e}_{3}\right)$ & $\hat{e}_{3}=R(\alpha, \beta, \gamma) \hat{z}$ \\
\hline
\end{tabular}




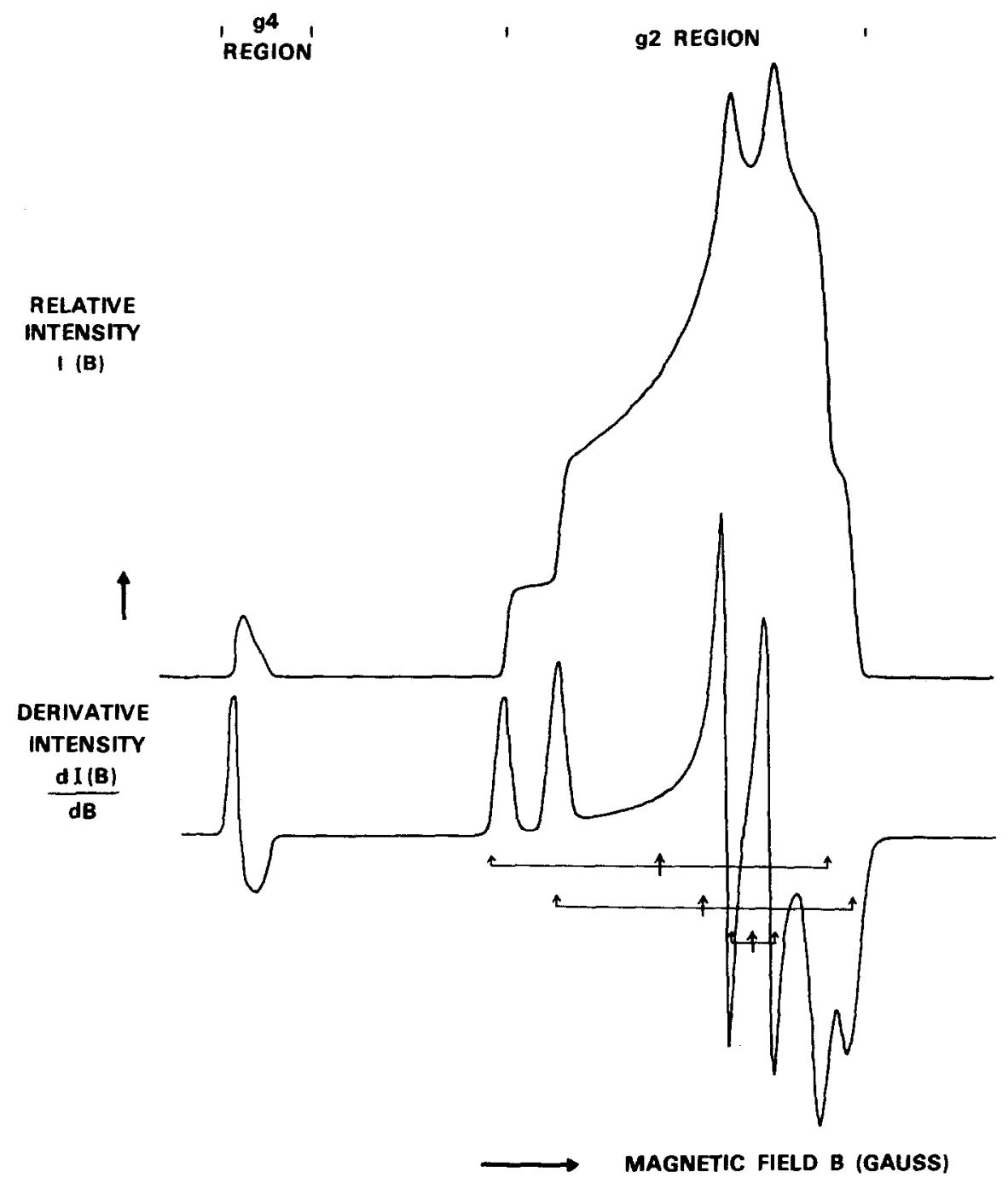

FIG. 3. Typical triplet spectra. The top figure is an absorption spectrum which includes $g 4$ and $g 2$ powder resonances. The bottom figure is the corresponding derivative spectrum. In the bottom figure are noted the principal splittings and centers of splitting.

is negative for all powder orientations; thus, the second-order term shifts the $g 4$ powder spectrum to lower fields, and, generally, the larger the principal values of $D$, then the larger the shifts. In addition, the second-order expression demonstrates that the higher the microwave frequency, the smaller the contributions from second-order corrections and the more the $g$ tensor dominates the turning points at $g 4$ (see Fig. 4).

The second-order correction can also profoundly affect lineshape. Generated $g 4$ spectra show that the $g 4$ lineshape is sensitive to variations in the Euler angles $\alpha, \beta$, and $\gamma$. Figure 5 demonstrates this variation with a few combinations of the three 


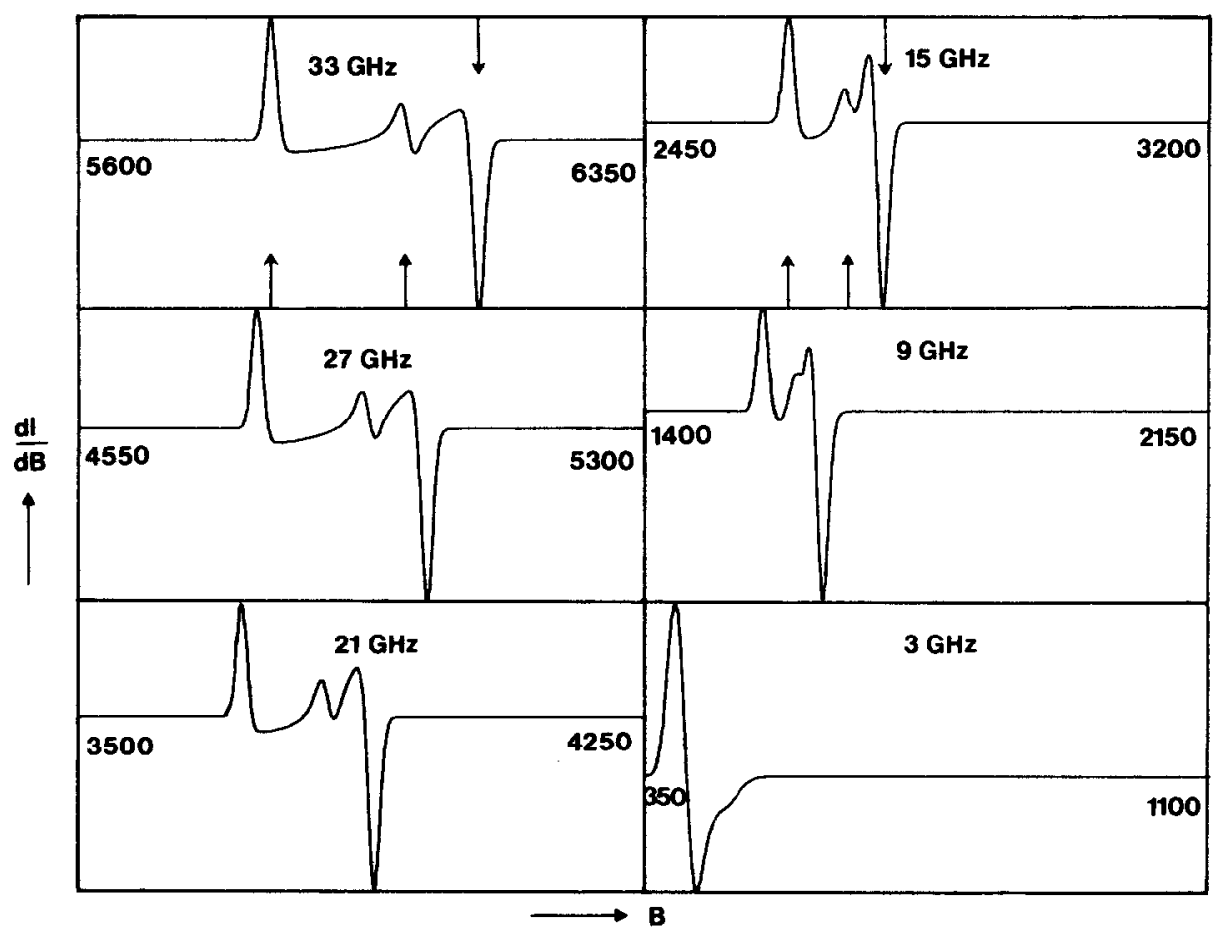

Fig. 4. Samplings of $g 4$ spectra versus frequency. All spectra have the same Hamiltonian parameters; only the frequency has been varied. All plots have the same gauss per-unit-length scale to show how the $g 4$ spectrum expands with increasing frequency. The end point fields are displayed on the plots.

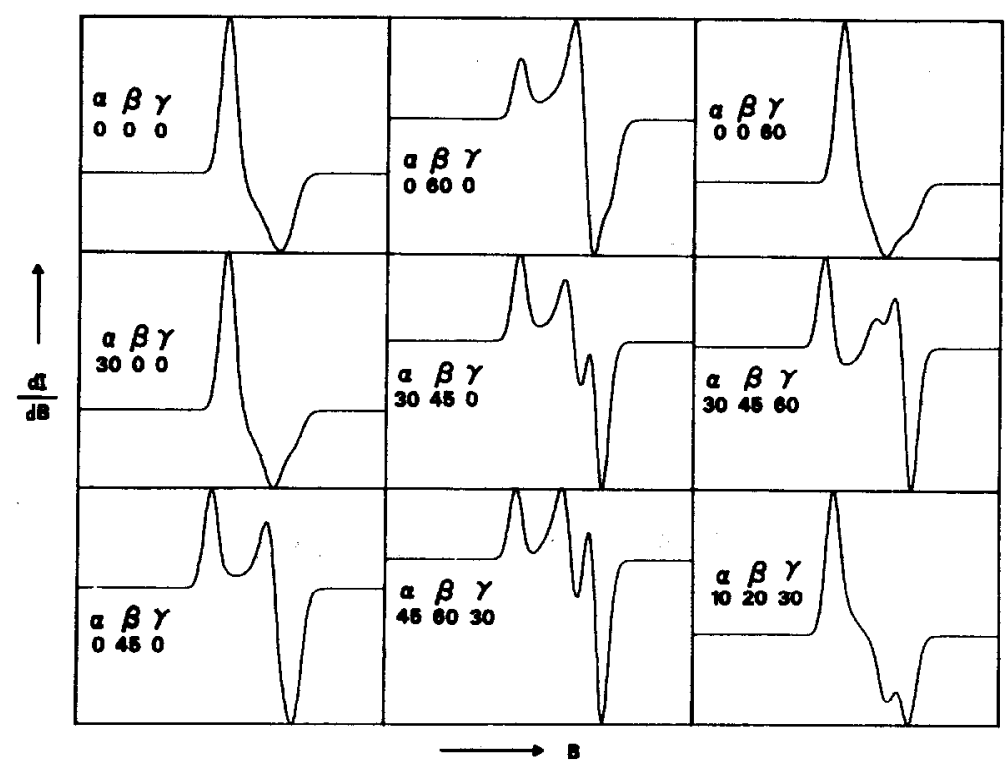

FIG. 5. Variation of $g 4$ spectra versus Euler angles. All parameters remain fixed except for Euler angles. 
angles. These variations are essentially the only visual handle one has to extract the Euler angles from the data independently of the other parameters. Variations in the Euler angles do not generally manifest themselves at $g 2$ as readily as at $g 4$.

Equation [79] also permits one to calculate a fairly accurate formula for the triplet $g$ values, if there exist $g 4$ data for at least two frequencies $\nu^{\mathrm{II}}>\nu^{\mathrm{I}}$. Suppose that the three principal turning points at $\nu^{\mathrm{l}}$ are $B_{3 i}^{\mathrm{I}}, i=x, y$, or $z$, with corresponding turning point fields at $\nu_{\mathrm{II}}$. The principal $g$ values, to second order, from Eq. [79] are

$$
g_{i} \stackrel{\substack{\text { second } \\ \text { order }}}{=} 1 / 2\left(\frac{h}{\beta}\right) \frac{B_{3 i}^{\mathrm{II}} \nu^{\mathrm{II}}-B_{3 i}^{\mathrm{I}} \nu^{\mathrm{I}}}{\left(B_{3 i}^{\mathrm{II}}\right)^{2}-\left(B_{3 i}^{\mathrm{I}}\right)^{2}}
$$

To show how well this approximate formula works, consider the simulated Q-band $(33 \mathrm{GHz})$ and the P-band (15 GHz) $g 4$ simulations of Fig. 4. The turning points measured from these two $g 4$ spectra are those listed in Table 3 . Table 4 illustrates the accuracy of the formula of Eq. [81] by comparing the $g$ values used to simulate the spectra with the $g$ values calculated from Eq. [81] using the numbers in Table 3.

Many laboratories do not have multifrequency capability, but when studying spinspin interaction it is almost mandatory to have data at at least two frequencies to be able to separate field-dependent interactions from field-independent interactions.

\section{RECIPE FOR FITTING DATA}

The qualitative behavior of triplet EPR spectra, as reviewed above, suggests a procedure or recipe for fitting EPR spectra suspected or known to be triplet data.

Step 1. (a) Find the first estimate for the principal $g$ values from the half-field data at two well-separated frequencies; or one might use Eq. [11] if one knows the two $g$ tensors and their relative orientations. Indeed, if the principal $g$ values from the data satisfy Eq. [11], this is strong evidence for a triplet interaction.

(b) Determine the principal splittings in gauss, and thus the first estimate of the principal values of the ZFS tensor.

(c) Determine the centers of splitting for the three principal splittings, and if the corresponding $g$ values for these centers are much different than the principal $g$ values determined in Step $1 \mathrm{a}$, then there clearly is a rotation $R(\alpha, \beta, \gamma) \neq 1$.

(d) Measure the linewidths of the turning points at $g 4$ and $g 2$. The $g 4$ linewidths should be approximately half the $g 2$ linewidths. The full linewidths at half maximum

TABLE 3

\begin{tabular}{cccc}
$\begin{array}{c}\text { Measured TuRNiNG PoINTS FROM } \\
\text { SimUlations FROM Fig. }\end{array}$ & $5^{a}$ \\
\hline & $B_{3 z}$ & $B_{3 y}$ & $B_{3 x}$ \\
\hline$(33 \mathrm{GHz}) \nu^{\mathrm{Il}}$ & 5856 & 6034 & 6131 \\
$(15 \mathrm{GHz}) \nu^{\mathrm{I}}$ & 2641 & 2719 & 2769 \\
\hline
\end{tabular}

a All fields in gauss. 
TABLE 4

SECOND-ORDER EXPRESSION FOR $g$ VALUES AND COMPARISON OF Calculated with Prescribed $g$ Values

\begin{tabular}{lccc} 
& $g_{z}$ & $g_{y}$ & $g_{x}$ \\
\hline For Fig. 4 value used in simulation & 2.010 & 1.950 & 1.920 \\
Calculated $g$ values from data in Table 3 & 2.009 & 1.949 & 1.920 \\
\hline
\end{tabular}

at $g 4$ are good first estimates for $\sigma_{x}$ and $\sigma_{z} ; \sigma_{y}$ requires more trial and error guess work.

Step 2. With the initial estimates for $g_{x}, g_{y}$, and $g_{z}, X, Y$, and $Z$, and $\sigma_{x}, \sigma_{y}$, and $\sigma_{z}$, simulate the $g 4$ data for various choices for the Euler angles $\alpha, \beta$, and $\gamma$.

Step 3. After obtaining a "reasonable" fit at $g 4$, with possibly some alterations in principal $g$ values and linewidths from the initial estimates, next simulate the $g 2$ region, refining the estimates for the ZFS parameters $X, Y$, and $Z$ during successive simulations.

Step 4. Work back and forth between the $g 2$ and $g 4$ refining parameters until one obtains the desired degree of accuracy in the fit.

The recipe for fitting given above, in some sense, constitutes a statement of the uniqueness of the fitting parameters; there are degeneracies in the Euler angles due to the symmetries of the trigonometric functions of the Euler rotation, but generally a fit is possible with all angles restricted to the first quadrant. The reader is referred to Ref. (11) for an example of the simulation of spin-coupled data using TRIPLET and the recipe above.

\section{CONCLUSIONS}

The calculation of EPR spectra from the triplet Hamiltonian is an interesting exercise in quantum mechanics. Closed-form expressions exist for many of the desired quantities; the $3 \times 3$ problem is tractable when compared to higher dimensions. The behavior of triplet EPR spectra plainly reflects the Hamiltonian parameters, if one knows how to look.

\section{APPENDIX: TRIPLET RENORMALIZATION}

All resonances have the form

$$
\Delta E=h \nu=\sqrt{3} m \cos \left(\theta+\delta_{r}\right) .
$$

where

$$
\delta_{r}= \pm \pi / 6 \text { and } 3 \pi / 6
$$

(see Eq. [48]). The corrections are

$$
\left|\frac{\partial \nu}{\partial B}\right|
$$


where

$$
h \frac{\partial \nu}{\partial B}=\sqrt{3}(d m / d B) \cos \left(\Theta+\delta_{r}\right)+\sqrt{3} m\left(-\sin \left(\Theta+\delta_{r}\right)\right) \cdot \frac{d \Theta}{d B} .
$$

The various derivatives one must insert into Eq. [A2] are

$$
\begin{aligned}
& \frac{d m}{d B}=-\frac{2}{3 m} \frac{d \theta}{d B}, \\
& \frac{d \theta}{d B}=\frac{1}{\sin 3 \Theta} \frac{b}{a}-\frac{2 b}{3 m^{2}}-2 D_{0} \frac{1}{a m} \frac{d a}{d B},
\end{aligned}
$$

and finally,

$$
\frac{d a}{d B}=-2 A_{r}(g \beta)
$$

Here,

$$
a=-A_{r}^{2}+t_{2} \quad \text { and } \quad-b=2 D_{0} A_{r}^{2}+t_{3} .
$$

The sequence of steps that a computer program must perform to calculate the corrections goes as follows:

$$
\begin{aligned}
& \text { Calculate (a) } \Theta \text { and } A_{r}, \text { (see Eqs. [34] and [51]). } \\
& \text { (b) } d a / d B \\
& \text { (c) } d \theta / d B \\
& \text { (d) } d m / d B \\
& \text { (e) } d \nu / d B
\end{aligned}
$$

\section{ACKNOWLEDGMENTS}

Thanks are due to $R$. H. Sands for suggesting the topic of this paper and to $W$. R. Dunham for discussions and criticisms of this work and to both individuals for their assistance in checking the accuracy of TRIPLET. Also, thanks go to M. Colby for processing the manuscript. This work was completed under the N.I.H. grant GM12176.

\section{REFERENCES}

1. S. P. MCGlynN, T. ASUMI, AND M. KinoshitA, "Molecular Spectroscopy of the Triplet State," Prentice-Hall, Englewood Cliffs, N.J., 1969.

2. G. M. MuHA, J. Magn. Reson. 49, 431 (1982)

3. M. DECORPS AND F. Genoud, J. Magn. Reson. 35, 247 (1979).

4. E. Wasserman, L. C. Snyder, and W. A. Yager, J. Chem. Phys. 41, 1763 (1964).

5. L. Banci, A. Becini, D. Galteschi, ANd C. Zanchini, J. Magn. Reson. 48, 9 (1982).

6. F. S. Ham, J. Chem. Phys. Solids 24, 1165 (1963).

7. T. Morrya, Phys. Rev. 120, 91 (1960).

8. "C. R. C. Standard Mathematical Tables," 23rd ed., p. 104, CRC Press, Cleveland, Ohio, 1975.

9. A. PAPoulis, "Probability, Random Variables, and Stochastic Processes," p. 126, McGraw-Hill, New York, 1965.

10. R. C. StevenSon, "Triplet State EPR: An Application to Three Iron-Sulfur Flavoproteins," Ph.D. Thesis, University of Michigan, Ann Arbor, 1982.

11. H. BEINERT, et al., "Flavins and Flavoproteins" (V. Massey and C. Williams, Eds.), p. 727 Elsevier/ North-Holland, Amsterdam/New York, 1982. 